\title{
Tighter Analysis of an Approximation for the Cumulative VRP*
}

\author{
Mauro Henrique Mulati ${ }^{1,2}$, Flávio Keidi Miyazawa ${ }^{1}$ \\ ${ }^{1}$ Institute of Computing - University of Campinas (UNICAMP) \\ Campinas - SP - Brazil \\ ${ }^{2}$ Department of Computer Science - Midwestern State University (UNICENTRO) \\ Guarapuava - PR - Brazil \\ $\{$ mhmulati,fkm\}@ic.unicamp.br
}

\begin{abstract}
We deal with the cumulative vehicle routing problem (VRP), a generalization of the capacitated VRP, which objective is to minimize the fuel consumption. Gaur et al. in 2013 gave a 4-approximation based on a well-known partition heuristic to the traveling salesperson problem (TSP). We present a tighter analysis obtaining a $\left(4-\frac{4}{3 s^{3} Q^{2}}\right)$-approximation, where $Q$ is the capacity of the vehicle and $s$ is a scaling factor. To the best of our knowledge, this is the best proved approximation for the cumulative VRP so far.
\end{abstract}

\section{Introduction and Previous Work}

The cumulative VRP was proposed by [Kara et al. 2008]. The objective is to minimize the fuel consumption, given that the fuel consumed by distance unit is linearly proportional to the total weight being carried (vehicle + load).

An instance of the cumulative VRP is given by what follows. A complete undirected graph $G(V, E)$ with vertices $V=\{0,1, \ldots, n\}$, where 0 is the depot and the other vertices are customers. There is an object of weight $w_{i} \in \mathbb{Q}_{>0}$ for each customer $i$, and we consider that $w_{0}=0$. Each edge $u v \in E$ has a length $d_{u v} \in \mathbb{Q}_{>0}$ satisfying the triangular inequality. An empty vehicle with capacity $Q \in \mathbb{Q}_{>0}$ and weight $W_{0} \in \mathbb{Q}_{>0}$ is initially located at the depot, and also, the weight of an object does not exceed $Q$. In a feasible solution $S$, we have that the only vehicle is repeatedly used in $k$ directed cycles, each one including the depot, to form a schedule that picks up the objects at the customers and drops them in the depot, visiting every customer exactly once. The objective is to obtain such a schedule that minimizes the fuel consumed.

Let $\mu>0$ be a constant that relates how much fuel is consumed by weight per distance unit. We define $a=\mu W_{0}$ and $b=\mu$. The fuel consumed by the vehicle to traverse the cycle $C_{j}$ is $f\left(C_{j}\right)=a\left|C_{j}\right|+b \sum_{i \in C_{j}} w_{i} d_{i}^{S}$, where $\left|C_{j}\right|$ is the length of the cycle $C_{j}$, and $d_{i}^{S}$ is the distance traveled by the vehicle carrying the object from being picked in the customer $i$ until being dropped at the depot in the schedule $S$. The fuel consumed by a vehicle to perform a schedule $S$ is $f(S)=a \sum_{j=1}^{k}\left|C_{j}\right|+b \sum_{i=1}^{n} w_{i} d_{i}^{S}$. Let $d_{i}$ be the shortest distance between vertex $i$ and the depot.

*Supported by CNPq (311499/2014-7 and 425340/2016-3), FAPESP (2015/11937-9), and SETI-PR / Fund. Araucária / CAPES.

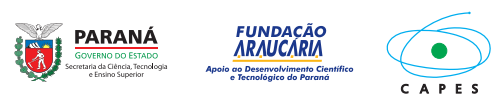


In this paper, we roughly follow the structure of [Gaur et al. 2013]. For our contribution, we use the theorems 1-4 from [Gaur et al. 2013] and the definitions presented below.

Theorem 1 ([Haimovich and Rinnooy Kan 1985, Gaur et al. 2013]). Let $C^{*}$ denote an optimal TSP tour of the graph $G(V, E)$. Then, the total distance traveled by a vehicle to bring all objects to the depot is at least $\max \left(\left|C^{*}\right|, 2 \frac{\sum_{i=1}^{n} w_{i} d_{i}}{Q}\right)$.

A subtour is a TSP tour that visits a subset of $V(G)$, and, when it is clear, we will use tour to denote it. W.l.o.g., consider that the vertices of a tour $C$ are numbered as $0,1, \ldots, n$ in the order that they appear in the sequence, and 0 is the depot. $|C|$ is the length of the tour $C$. By a cluster $[i, j]$ we mean a set of a sequence of vertices in the tour $C$ from $i$ to $j$, with the extremes included. Considering $k \geq 2$, and $1<x_{1}<x_{2}<\cdots<x_{k-1} \leq n$, a cluster partition denoted by $P=\left[1, x_{1}, x_{2}, \ldots, x_{k-1}, n\right]$ of tour $C$ is a decomposition of $C$ into the $k$ clusters $\left[1, x_{1}\right],\left[x_{1}, x_{2}\right], \ldots,\left[x_{k-1}, n\right]$. From a cluster partition $P$ of $C$, we are able to construct $k$ subtours $C_{1}, \ldots, C_{k}$ such that: to traverse the subtour $C_{j}$, the vehicle begins at the depot, visits the vertices of $C_{j}$ in increasing order, and ends in the depot. The length of $P$ is given by $l(P)=\left|C_{1}\right|+\cdots+\left|C_{k}\right|$.

Theorem 2 ([Altinkemer and Gavish 1987, Gaur et al. 2013]). Let an integer $s>0$ be a scaling factor in a way that $s w_{i}$, for every $i \in V, s W_{0}$, and $s Q$ are positive integers, such that $s w_{i} \leq s Q$ for every $i \in V$. Let $C$ be a TSP tour of $G$ and let $Q$ be the vehicle capacity. Then, there exists a cluster partition $P$ of $C$ with total length at most $4 \frac{\sum_{i=1}^{n} w_{i} d_{i}}{Q}+\left(1-\frac{2}{s Q}\right)|C|$.

Theorem 3 ([Gaur et al. 2013]). Let $C^{*}$ be an optimal TSP tour, and let $Q$ be the capacity of the vehicle. Then, the minimum fuel consumed by the vehicle to bring all objects to the depot is at least $a \cdot \max \left(\left|C^{*}\right|, 2 \frac{\sum_{i=1}^{n} w_{i} d_{i}}{Q}\right)+b\left(\sum_{i=1}^{n} w_{i} d_{i}\right)$.

Theorem 4 ([Gaur et al. 2013]). Let $\beta>0$ be a positive rational number, $C$ be a TSP tour, and assume that the vehicle has infinite capacity. Then, there exists a cluster partition $P=\left[1, x_{1}, x_{2}, \ldots, x_{k-1}, n\right]$ of $C$ with total fuel consumption at most $\left(1+\frac{2}{\beta}\right) b\left(\sum_{i=1}^{n} w_{i} d_{i}\right)+\left(1+\frac{\beta}{2}\right) a|C|$.

\section{Our Contribution}

We provide, in theorems 5 and 6, a refined analysis of the algorithm of [Gaur et al. 2013], showing a tighter approximation ratio than the one presented by them.

Theorem 5 (From [Gaur et al. 2013] with a tighter bound). Let $\beta>0$ be a positive rational number, $C$ be a TSP tour, and $Q$ be the vehicle capacity. Then, there exists a cluster partition $P=\left[1, x_{1}, x_{2}, \ldots, x_{k-1}, n\right]$ of $C$ with total fuel consumption at most $\left(1+\frac{2}{\beta}\right) b\left(\sum_{i=1}^{n} w_{i} d_{i}\right)+\left(1+\frac{\beta}{2}\right) a|C|+4 a \frac{\sum_{i=1}^{n} w_{i} d_{i}}{Q}-2 a \frac{\sum_{j=1}^{k}\left|C_{j}\right|}{s Q}$.

Proof. Considering infinite capacity, there exists a cluster partition $P$ of tour $C$ with fuel consumption $f(P)$ with an upper bound given by Theorem 4 . Let $C_{1}, C_{2}, \ldots, C_{k}$ be the subtours corresponding to the cluster partition $P$. Let $W_{j}$ be the total weight of the objects picked by the vehicle in the subtour $C_{j}$. If $W_{j} \leq Q$, then $C_{j}$ satisfies the capacity restriction and, consequently, we keep the cluster corresponding to the subtour $C_{j}$ with fuel consumption $f\left(C_{j}\right)$ unchanged. On the other hand, assume that $W_{j}>Q$ : by 
Theorem 2 there exists a refined cluster partition $P_{j}$ of $C_{j}$ such that the total weight of the objects in each cluster of $P_{j}$ is at most $Q$, and there exists an upper bound on its length.

Now, given a subtour $C_{j}$, we will give an upper bound on the fuel consumption $f\left(P_{j}\right)$. W.l.o.g., we assume that the fuel consumption $f\left(C_{j}\right)$ of the subtour $C_{j}$ is obtained by a traversal in clockwise order (the reversed case is symmetric). Consider that the vehicle traverses each subtour $C_{j l}, 1 \leq l \leq k_{j}$ in the partition $P_{j}$. We have that $V_{j}$ is the set of vertices of the tour $C_{j}$. Consider that, for each vertex $i \in V_{j}$, we have $d_{i}^{C_{j}}$ that represents the distance traveled by the vehicle from picking object $i$ to dropping it at the depot in tour $C_{j}$, and analogously, $d_{i}^{P_{j}}$ represents the respective distance for the cluster partition $P_{j}$. We have that $d_{i}^{P_{j}} \leq d_{i}^{C_{j}}$, because the cluster partition $P_{j}$ is a refinement of the tour $C_{j}$. Thus we can write $f\left(P_{j}\right)=\sum_{l=1}^{k_{j}} f\left(C_{j l}\right)=\sum_{l=1}^{k_{j}}\left(a\left|C_{j l}\right|+b \sum_{i \in C_{j l}} w_{i} d_{i}^{P_{j}}\right)=$ $a \sum_{l=1}^{k_{j}}\left|C_{j l}\right|+b \sum_{i \in C_{j}} w_{i} d_{i}^{P_{j}} \leq a \cdot l\left(P_{j}\right)+b \sum_{i \in C_{j}} w_{i} d_{i}^{C_{j}} \leq 4 a \frac{\sum_{i \in C_{j}} w_{i} d_{i}}{Q}+a\left|C_{j}\right|-$ $a\left|C_{j}\right| \frac{2}{s Q}+b \sum_{i \in C_{j}} w_{i} d_{i}^{C_{j}}=f\left(C_{j}\right)+4 a \frac{\sum_{i \in C_{j}} w_{i} d_{i}}{Q}-a\left|C_{j}\right| \frac{2}{s Q}$, where we used the upper bound on $l\left(P_{j}\right)$ given by Theorem 2 .

We consider that $P^{\prime}$ is the final cluster partition that includes all the clusters, the ones unchanged as well as the ones refined. Thus, the total fuel consumption is given by $f\left(P^{\prime}\right)=\sum_{j=1}^{k} f\left(P_{j}\right) \leq \sum_{j=1}^{k}\left(f\left(C_{j}\right)+4 a \frac{\sum_{i \in C_{j}} w_{i} d_{i}}{Q}-a\left|C_{j}\right| \frac{2}{s Q}\right)=\sum_{j=1}^{k} f\left(C_{j}\right)+$ $4 a \frac{\sum_{j=1}^{k} \sum_{i \in C_{j}} w_{i} d_{i}}{Q}-2 a \frac{\sum_{j=1}^{k}\left|C_{j}\right|}{s Q}=f(P)+4 a \frac{\sum_{j=1}^{n} w_{i} d_{i}}{Q}-2 a \frac{\sum_{j=1}^{k}\left|C_{j}\right|}{s Q}$. By the upper bound of Theorem 4 on $f(P)$, we have that $P^{\prime}$ is a cluster partition satisfying the theorem.

Lemma 1. Let $C^{*}$ be an optimal TSP tour in a complete graph $G(V, E)$ with weight function $d$ that are part of an instance of the capacitated VRP. Then, $\left|C^{*}\right| \leq 2 \sum_{i=1}^{n} d_{i}$.

Proof. Recall that $|V|=n+1$, with the vertices numbered from 0 (depot) to $n$. By definition $\left|C^{*}\right|=\sum_{u v \in E\left(C^{*}\right)} d_{u v} \leq \sum_{u v \in E\left(C^{*}\right)}\left(d_{u}+d_{v}\right)=\sum_{i \in V\left(C^{*}\right)} 2 d_{i}=2 \sum_{i=0}^{n} d_{i}=$ $2 \sum_{i=1}^{n} d_{i}$, where we used: that $d_{u v} \leq d_{u 0}+d_{0 v}$ for $u v \in E\left(C^{*}\right)$ by the triangular inequality, the definition that $d_{i 0}=d_{0 i}=d_{i}$ for every $i \in V$, the fact that each vertex $i$ is an extreme of exactly two edges of $C^{*}$, and the definition $d_{0}=0$.

Theorem 6. There exists a factor $4-\frac{4}{3 s^{3} Q^{2}}$ polynomial-time approximation algorithm for the cumulative VRP.

Proof. Given as input an instance of cumulative VRP as previously described, consider the algorithm with the steps: (1) compute a tour $C$ of $G$ by the well-known Christofides' algorithm [Gaur et al. 2013], which guarantees that $|C| \leq \frac{3}{2}\left|C^{*}\right|$; (2) compute a cluster partition $P^{*}$ of tour $C$ with optimal fuel consumption by a DP algorithm in time $O\left(n^{2}\right)$ as done in [Gaur et al. 2013]; and (3) return the subtours $C_{1}^{*}, C_{2}^{*}, \ldots, C_{k}^{*}$ of $P^{*}$.

In this algorithm, we optimally calculate $P^{*}$ of $C$ in polynomial time. The analysis is being made over a heuristic algorithm that also calculates a cluster partition $P$ of a tour, thus we can state that $f\left(P^{*}\right) \leq f(P)$. Let $S^{*}$ be an optimal routing scheduling in fuel consumption. By theorems 5 and 3 , we have the ratio

$$
\frac{f\left(P^{*}\right)}{f\left(S^{*}\right)} \leq \frac{\left(1+\frac{2}{\beta}\right) b\left(\sum_{i=1}^{n} w_{i} d_{i}\right)+\left(1+\frac{\beta}{2}\right) a|C|+4 a \frac{\sum_{i=1}^{n} w_{i} d_{i}}{Q}-2 a \frac{\sum_{j=1}^{k}\left|C_{j}\right|}{s Q}}{a \max \left(\left|C^{*}\right|, 2 \frac{\sum_{i=1}^{n} w_{i} d_{i}}{Q}\right)+b \sum_{i=1}^{n} w_{i} d_{i}}
$$




$$
\begin{aligned}
& \leq \frac{4\left(a\left(\frac{\left|C^{*}\right|}{2}+\frac{2}{2} \frac{\sum_{i=1}^{n} w_{i} d_{i}}{Q}\right)+b \sum_{i=1}^{n} w_{i} d_{i}\right)-2 a \frac{\sum_{j=1}^{k}\left|C_{j}\right|}{s Q}}{a \max \left(\left|C^{*}\right|, 2 \frac{\sum_{i=1}^{n} w_{i} d_{i}}{Q}\right)+b \sum_{i=1}^{n} w_{i} d_{i}} \\
& \leq 4-\frac{2 a \sum_{j=1}^{k}\left|C_{j}\right|}{s Q\left(a \max \left(\left|C^{*}\right|, 2 \frac{\sum_{i=1}^{n} w_{i} d_{i}}{Q}\right)+b \sum_{i=1}^{n} w_{i} d_{i}\right)} \\
& \leq 4-\frac{2 a \max \left(\left|C^{*}\right|, 2 \frac{\sum_{i=1}^{n} w_{i} d_{i}}{Q}\right)}{s Q\left(a \max \left(\left|C^{*}\right|, 2 \frac{\sum_{i=1}^{n} w_{i} d_{i}}{Q}\right)+b \sum_{i=1}^{n} w_{i} d_{i}\right)} \\
& =4-\frac{2 \mu W_{0} \max \left(\left|C^{*}\right|, 2 \frac{\sum_{i=1}^{n} w_{i} d_{i}}{Q}\right)}{s Q\left(\mu W_{0} \max \left(\left|C^{*}\right|, 2 \frac{\sum_{i=1}^{n} w_{i} d_{i}}{Q}\right)+\mu \sum_{i=1}^{n} w_{i} d_{i}\right)} \\
& \leq 4-\frac{2 W_{0} \max \left(\left|C^{*}\right|, 2 \frac{\sum_{i=1}^{n} w_{i} d_{i}}{Q}\right)}{s Q\left(s W_{0} \max \left(\left|C^{*}\right|, 2 \frac{\sum_{i=1}^{n} w_{i} d_{i}}{Q}\right)+s W_{0} \sum_{i=1}^{n} s w_{i} d_{i}\right)} \\
& =4-\frac{2 \max \left(\left|C^{*}\right|, 2 \frac{\sum_{i=1}^{n} w_{i} d_{i}}{Q}\right)}{s^{2} Q\left(\max \left(\left|C^{*}\right|, 2 \frac{\sum_{i=1}^{n} w_{i} d_{i}}{Q}\right)+\sum_{i=1}^{n} s w_{i} d_{i}\right)} \\
& \leq 4-\frac{4 \frac{\sum_{i=1}^{n} w_{i} d_{i}}{Q}}{3 s^{3} Q \sum_{i=1}^{n} w_{i} d_{i}}=4-\frac{4}{3 s^{3} Q^{2}} \cdot
\end{aligned}
$$

To obtain (1), we chose $\beta=\frac{2}{3}$ and used that $|C| \leq \frac{3}{2}\left|C^{*}\right|$. We used Theorem 1 to get (2). To obtain (3), recall that $a=\mu W_{0}$ and $b=\mu$. In (4), we made use of the fact that $s$ and $s W_{0}$ are integers. To obtain (5), we deal with the absolute value of the fraction: we kept or lower the numerator; and we majored the denominator applying the fact that $\left|C^{*}\right| \leq 2 \sum_{i=1}^{n} d_{i} \leq 2 \sum_{i=1}^{n} s w_{i} d_{i}$ as Lemma 1 states and as $s w_{i}$ are integers for every $i \in V$, and the fact $2 \frac{\sum_{i=1}^{n} w_{i} d_{i}}{Q}=2 \frac{\sum_{i=1}^{n} s w_{i} d_{i}}{s Q} \leq 2 \sum_{i=1}^{n} s w_{i} d_{i}$, as $s Q$ is integer.

\section{References}

Altinkemer, K. and Gavish, B. (1987). Heuristics for unequal weight delivery problems with a fixed error guarantee. Operations Research Letters, 6(4):149 - 158.

Gaur, D. R., Mudgal, A., and Singh, R. R. (2013). Routing vehicles to minimize fuel consumption. Operations Research Letters, 41(6):576 - 580.

Haimovich, M. and Rinnooy Kan, A. H. G. (1985). Bounds and heuristics for capacitated routing problems. Mathematics of Operations Research, 10(4):527-542.

Kara, I., Kara, B. Y., and Yetiş, M. K. (2008). Cumulative Vehicle Routing Problems, pages 85-98. InTech Education and Publishing KG, Vienna, Austria. 